\title{
Efecto de sistemas silvopastoriles en la producción y composición de la leche bajo condiciones del valle medio del rio sinú, Colombia
}

\section{Effect of silvopastoral systems on production and milk composition under sinu river valley conditions, Colombia}

\author{
BARRAGÁN HERNÁNDEZ WILSON ${ }^{1}$ M.Sc, MAHECHA-LEDESMA LILIANA² Ph.D, \\ CAJAS-GIRÓN YASMIN ${ }^{1}$ Ph.D.
}

${ }^{1}$ Corporación Colombiana de Investigación Agropecuaria - CORPOICA. Centro de Investigación Turipaná, Montería - Cereté, Córdoba, Colombia. ${ }^{2}$ Universidad de Antioquia, Facultad de Ciencias Agrarias. Grupo de Investigación en Ciencias Agrarias (GRICA) Producción Animal Sostenible. Medellín, Antioquia, Colombia

\section{Keywords:}

Forage,

meadow,

trees,

grasses,

lactation,

model Wood.

Palabras Clave:

Arboles,

forraje,

gramíneas,

lactancia

modelo de Wood,

pradera

\section{Abstract}

The aim of this study was evaluated the effect of three silvopastoral models in production and compositional quality of milk, compared with a treeless prairie. Animals were used from a cross $\mathrm{RHC}$ with an average weight of $436 \pm 64.2 \mathrm{~kg}$ and $56 \pm 28$ days in milk. The animals were subjected to a daily routine of milking in which production was recorded and samples were taken for analysis of compositional quality of milk. The results indicated no treatment effect on dry matter production ( $p>0.05)$, contrary to this, the treatment effect was recorded in the percentage of PC for the grass Panicum maximum, indicating that with increasing tree cover increased to $12.6 \% \mathrm{PC}$, bought with $10 \%$ in the treatment without trees. There were no significant differences $(p>0.05)$ between treatments for Wood model parameters, and time to peak production at peak production, but appeared significant $(p<0.05)$ in milk production per ha, indicating that treatment silvopastoral including shrubs, recorded the highest milk yield ha-1 day-1 with a value of $12.8 \mathrm{I}$. No significant effect of treatment on milk compositional quality assessed. The results indicated that the model that includes shrubs silvopastoral resulted in increased efficiency of production capacity per unit area, which was influenced by increased forage production and higher load capacity.

\section{Resumen}

El presente trabajo evaluó el efecto de tres arreglos silvopastoriles conformados por pastoArbusto, pasto-arbóreas y pasto-arbusto-arbóreas, en la producción y calidad composicional de la leche, comparados con una pradera sin árboles. Se emplearon animales provenientes de un cruce $\mathrm{RHC}$ con un peso promedio de $436 \pm 64.2 \mathrm{~kg}$ y $56 \pm 28$ días en lactancia. Los animales fueron sometidos a una rutina diaria de ordeño registrando la producción de leche y se tomaron muestras para análisis de calidad composicional de la misma. Los resultados no indicaron efecto del tratamiento en la producción de materia seca $(p>0.05)$, contrario a esto, se registró efecto del tratamiento en el porcentaje de PC para la gramínea Panicum maximum, indicando que a medida que aumenta la cobertura arbórea se incrementó la PC hasta $12.6 \%$, comprada con $10 \%$ en el tratamiento sin árboles. No se registraron diferencias significativas $(p>0.05)$ entre los tratamientos para los parámetros de modelo de Wood, producción al pico y tiempo al pico de producción en la lactancia. Sin embargo, se presentó efecto significativo $(p<0.05)$ de la producción de leche por ha, indicando que el tratamiento silvopastoril que incluye especies arbustivas, registró la mayor producción de leche ha-1 $\mathrm{dia}^{-1}$ con un valor de $12.8 \mathrm{I}$. No se presentaron efectos significativos del tratamiento sobre la calidad composicional de la leche. Los resultados indicaron que el arreglo silvopastoril que incluye arbustos resultó en mayor producción de leche por unidad de superficie, lo cual pudo estar influenciado por la mejor calidad de la dieta ofertada y mayor capacidad de carga. 


\section{Introducción}

La ganadería en la región Caribe representa un renglón importante en la economía regional (OBSERVATORIO DE CARIBE COLOMBIANO, 2006), aportando el $55 \%$ del volumen de leche fresca y el $60 \%$ del volumen de carne que se comercializa en el país (TATIS y BOTERO, 2005). Sin embargo, pese a la importancia de la actividad en la región, el sistema de producción doble propósito evidencia bajos indicadores productivos y reproductivos (PÉREZ et al., 1998).

Estudios desarrollados por CAJAS-GIRÓN (2002) identificaron que bajo la región Caribe, la ganadería enfrenta serios limitantes para su eficiencia como lo son la disminución en la producción y calidad del forraje durante el período seco, los cuales pueden ser hasta del 50 y $70 \%$ respectivamente. Adicionalmente, la degradación de suelos y praderas ha ganado espacios en las áreas bajo uso en ganadería, con niveles de 73, 69 y $94 \%$ para los departamentos de Córdoba, Sucre y Atlántico respectivamente (CAJAS-GIRÓN et al., 2010), lo cual se constituye en otro factor para la ineficiencia técnica y biológica en el sistema de producción.

Diferentes autores han documentado los efectos de los sistemas silvopastoriles en la relación suelo-planta-animal, incrementando el contenido de nutrientes en el suelo (NAIR et al., 1999; SCHROTH, 2003), afectando las características bromatológicas y estructurales de las gramíneas (WILSON, 1996; WONG, 1991) y mejorando la calidad de la dieta consumida (PEZO E IBRAHIM, 1998; CAJAS-GIRÓN et al., 2002; PINTO et al., 2004), así como también, reduciendo el efecto del estrés calórico en los animales (BETANCOURT et al.,2003; GARCÍA, 2010), por lo que se considera que este tipo de sistemas de producción puede ser un componente importante del desarrollo productivo de la ganadería bajo condiciones de trópico.

Estudios que relacionan el efecto de los sistemas silvopastoriles en la producción de leche han reportado que este tipo de sistemas de producción incrementa el volumen en litros por animal (SOUZA 2002; CAJAS-GIRON, 2002; BETANCOURT et al., 2003; HERNÁNDEZ y PONCE, 2004; RONCALLO et al., 2009), así como también, mejora la calidad composicional de la leche (HERNÁNDEZ y PONCE, 2004; RONCALLO et al., 2009).

El objetivo del presente trabajo fue valorar el efecto de tres arreglos silvopastoriles en la producción y composición de la leche, comparado con una pradera sin árboles bajo condiciones del Valle del Sinú.

\section{Materiales y Métodos}

Área de estudio y diseño del experimento: La evaluación se llevó a cabo en el centro de investigación Turipaná, adscrito a la Corporación colombiana de investigación agropecuaria (CORPOICA), localizado en Cereté, Córdoba, Colombia $\left(8^{\circ} 51^{\prime} \mathrm{N}, 75^{\circ} 49^{\prime} \mathrm{W}\right.$, altitud de 18 m.m.s.n.m). La zona presenta dos periodos climáticos definidos, un periodo de lluvias de mayo a noviembre y un periodo seco de diciembre a abril. La precipitación, promedia anual es de $1.380 \mathrm{~mm}$, con una temperatura media anual de $28^{\circ} \mathrm{C}$. Se registra una evapotranspiración potencial anual de $1.240 \mathrm{~mm}$ y humedad relativa de $81 \%$ (Estación climatológica CORPOICA-Turipaná). Según la clasificación de Holdrige (HOLDRIDGE et al., 1971) la zona se clasifica ecológicamente como bosque seco tropical (bs-T).

En el año 1998 se establecieron cuatro tipos de sistemas silvopastoriles de diferente estructura y complejidad más una pradera sin árboles (control). Para efectos de esta investigación solamente fueron usados tres tratamientos silvopastoriles. Los tratamientos utilizados estuvieron compuestos por tres distintos estratos: pasturas, arbustos y árboles (productores de frutos). El tratamiento control (Pasto) estuvo conformado solo por pasturas (Panicum maximum, Dichanthium aristatum y Cynodon nlemfuensis), el primer sistema silvopastoril presentó solamente arbustos (p-Arbust), el segundo arreglo incluyó un estrato de árboles ( $p$-Arbor) y el último arreglo silvopastoril (p-Arbust-Arbor), la combinación de pasturas, arbustos y árboles. Inicialmente las densidades utilizadas en el establecimiento fueron 625 y 159 arbustos ha ${ }^{-1}$. en los arreglos silvopastoriles p-Arbust y p-Arbor, respectivamente. Para el caso del sistema silvopastoril p-Arbust-Abror, se 
utilizó un método de sustitución del $25 \%$ de la población de arbustos por árboles para obtener tres estratos, en ese orden de ideas, en este tratamiento la densidad de arbustos fue de 468 ha-1., y la de árboles de 159 ha-1. $^{-1}$ En el año 2004, se realizó una entresaca de especies arbóreas, disminuyendo de 159 árboles ha ${ }^{-1}$ (distancia de siembra de $8 \times 8$ metros) a 39 árboles ha- 1 . (Distancia de siembra $16 \times 16$ metros). Para el estrato arbustivo se utilizaron las especies Leucaena leucocephala y Crecentia cujete, y para el estrato arbóreo las especies fueron Guazuma ulmifolia, Cassia grandis y Albizia saman (Tabla 1). Cada tratamiento estuvo constituido de 2 hectáreas (100 metros de ancho por 200 metros de largo), para un total de 6 hectáreas efectivas para pastoreo en las tres repeticiones.

Se utilizó un diseño de bloques completos al azar con tres repeticiones. Cada tratamiento estuvo constituido por 2 hectáreas, generando un área efectiva de pastoreo por tratamiento de 6 hectáreas.

Animales evaluados: Se emplearon animales provenientes de un cruce Romosinuano (50\%), Holstein $(25 \%)$ y Cebú Comercial $(25 \%)$ con un peso promedio de $436 \pm 64.2 \mathrm{~kg}$ y $56 \pm 28$ días en lactancia.
Los animales estuvieron sometidos a una rutina diaria de ordeño mecánico iniciada a las 6 a.m (ordeño único), regresando al potrero a las 8 a.m. Se realizó pastoreo rotacional (mediante cinta eléctrica) con 5 franjas por tratamiento y un área de pastoreo de $4000 \mathrm{~m}^{2}$ por cada franja. Cada franja de pastoreo tuvo un periodo de ocupación y descanso de 2 y 28 días respectivamente.

Los terneros fueron manejados mediante amamantamiento restringido. La cría permanecía con la madre durante el ordeño para realizar el apoyo posteriormente se encuentra con la madre para tomar la leche residual. Después de este proceso, el ternero es apartado a un potrero con disponibilidad de forraje, sal y agua a voluntad, y solo se encentró con la madre las horas de la tarde (2:00 p.m) para ser amamantado, ingiriendo el volumen total de leche producido desde la mañana. Las crías mayores a cuatro meses solo consumían la leche residual después del ordeño.

Registro de la información: En cada tratamiento se determinó la producción de materia seca por hectárea con una frecuencia mensual (desde febrero de 2011 a diciembre de 2012) empleando la metodología de disponibilidad por frecuencia descrita por HOYOS et al. (1995), de igual forma se tomó una muestra de la

Tabla 1. Complejidad estructural y diversidad vegetal para cada uno de los modelos silvopastoriles evaluados y para la pradera sin árboles

\begin{tabular}{|c|c|c|c|}
\hline Tratamiento & Descripción & Estratos & Especies (\% composición herbácea) \\
\hline Pasto & Pastura & 1 & $\begin{array}{l}\text { Estrato herbáceo }=\text { Panicum maximum }(40 \%) \text {, Dichanthium aristatum }(34 \%) \text {, } \\
\text { Cynodon nlemfuensis }(13 \%) \text {, otras especies }(5 \%) \text { y leguminosas herbáceas }(8 \%)\end{array}$ \\
\hline p-Arbust & $\begin{array}{l}\text { Pastura }+ \\
\text { Arbustos } \\
\text { productores de } \\
\text { hojas }\end{array}$ & 2 & $\begin{array}{l}\text { Estrato herbáceo=Panicum maximum }(49 \%) \text {, Dichanthium aristatum }(25 \%) \text {, } \\
\text { Cynodon nlemfuensis }(10) \text {, otras especies }(10 \%) \text { y leguminosas herbáceas }(6 \%) \\
\text { Estrato arbustivo= Leucaena leucocephala y Crecentia cujete. } \\
\text { Densidad de arbustos }=625 \text { árboles } \text { ha }^{-1} \text {. } \\
\text { Marco de siembra } 4 \times 4 .\end{array}$ \\
\hline p-Arbor & Pasto + Arboles & 2 & $\begin{array}{l}\text { Estrato herbáceo=Panicum maximum }(68 \%) \text {, otras espécies }(19 \%) \\
\text { Estrato arbórero= Cassia gradis, Albizia saman y Guazuma ulmifolia. } \\
\text { Densidad de árboles }=39 \text { árboles } \text { ha }^{-1} \text {. } \\
\text { Distancia de siembra }=16 \times 16\end{array}$ \\
\hline p-Arbust-Arbor & $\begin{array}{l}\text { Pasto + Arbustos } \\
+ \text { Arboles }\end{array}$ & 3 & $\begin{array}{l}\text { Estrato herbáceo = Panicum maximum }(60 \%) \text {, otras especies }(15 \%) \\
\text { Estrato arbustivo= Leucaena leucocephala y Crecentia cujete. } \\
\text { Estrato arbóreo= Cassia gradis, Albizia saman y Guazuma ulmifolia } \\
\text { Densidad de arbustos = } 468 \text { arbustos ha }{ }^{-1} . \\
\text { Densidad de árboles }=39 \text { árboles ha }{ }^{-1} \text {. } \\
\text { Distancia de siembra arbustos }=4 \times 4 \text { metros. } \\
\text { Distancia de siembra de árboles sustituidos por arbustos = } 16 \times 16 \text { metros. }\end{array}$ \\
\hline
\end{tabular}


gramínea Panicum maximum cv. Mombasa, por tener mayor participación en la composición herbácea de la pradera, y de las arbustivas Leucaena leucocephala y Crescetia cujete en cada tratamiento, con la cual se determinó la composición bromatológica siguiendo los lineamientos descritos por la AOAC (1990), para cuantificar proteína (Kjendal), Grasa (Soxhlet) y ceniza (combustión completa), las estimaciones de FDN y FDA según lo descrito por Van Soest y Robertson (1981) y la digestibilidad in situ a través de la técnica de la bolsa de nylon.

Con los resultados obtenidos en la estimación de la materia seca, se determinó la carga animal, la cual se ajustó con el uso de animales volantes durante todo el experimento.

Los datos de producción de leche se colectaron de forma continua desde marzo de 2010 hasta diciembre de 2011, mediante ordeño mecánico (Alfamatic VP-76, con capacidad para 12 puestos). A cada animal se le registró la producción individual del volumen de leche en litros, en un único ordeño.

La evaluación de la calidad composicional de la leche se realizó en un periodo de 5 meses (agosto a diciembre de 2012), con una frecuencia de registro de tres veces por semana. Esta variable se determinó mediante muestreo individual a cada animal y posterior análisis en laboratorio CORPOLAC (CORPOICA), utilizando un Analizador de Leche (Milkoscan FT 120), Marca FOSS.

Análisis Estadístico: Los datos colectados fueron almacenados en una hoja de cálculos de Microsoft Excel® para su organización y análisis. En el caso de los registros obtenidos para la producción de forraje y calidad bromatológica de la gramínea $P$. maximum y arbustivas $L$. leucocephala y $C$. cujete se utilizó un diseño de bloques completos al azar (DBCA) con 4 tratamientos (Pasto, p-Arbust, p-Arbor y p-Arbust-Arbor) y 3 repeticiones. El factor de bloqueo se consideró por variaciones en la capacidad de drenaje del área utilizada, la cual se caracterizó con buen drenaje, drenaje moderado y mal drenaje. La hipótesis alterna esperada fue obtener efecto de los sistemas silvopastoriles en la producción de forraje por hectárea y cambios en la calidad bromatológica del componente herbáceo y arbustivo.
Los resultados obtenidos en producción de leche de manera individual, fueron analizados empleado el modelo de curva de lactancia de Wood (1967) mediante modelo de regresión no lineal empleando el procedimiento NLIN del paquete estadístico SAS (versión 9.2). En el procedimiento anterior, fueron obtenidos los parámetros de tiempo al pico de lactancia, producción en el pico y producción total, los cuales fueron analizados empleando un ANOVA, que consideró como unidades experimentales las vacas asignadas a cada tratamiento, y el tratamiento evaluado como efecto fijo en el modelo. Para este análisis se empleó el procedimiento GLM del paquete estadísticos SAS, considerando como error alfa 0.05 y el test TUKEY para comparación de medias.

$y_{t}=\beta_{0} t^{\beta_{1}} \exp \left(-\beta_{2} t\right)$ Modelo de Wood

$y_{t}=$ Producción de leche en un tiempo determinado. $\beta_{0}=$ Producción de leche al inicio de la lactancia. $\beta_{1}=$ Parámetro que explica la pendiente de la curva.

$\beta_{2}=$ Parámetro que explica la desaceleración de la curva de lactancia.

Para el análisis de los datos obtenidos en la evaluación en la calidad bromatológica de la pastura se utilizó un ANOVA mediante el procedimiento MIXED, considerando como efectos fijos el tratamiento, y como efectos aleatorios el factor de bloqueo y el error experimental. En el caso de la producción de forraje $\left(\mathrm{kg}_{\mathrm{g}}\right.$ de $\left.\mathrm{MS} \mathrm{ha}^{-1}\right)$ y leche $\left(\mathrm{l} \mathrm{ha}^{-1}\right)$, se empleó un modelo mixto con arreglo de medidas repetidas, el cual consideró como efectos fijos el tratamiento evaluado y el tiempo de evaluación (mes de muestreo), y como efectos aleatorios, el factor de bloqueo y el error asociado al diseño experimental. Para esta evaluación, se modeló la estructura de varianzas que mejor explicó la posible correlación entre las observaciones, con base en el estadístico BIC. Para todos los casos se empleó el paquete estadístico SAS versión. 9.2 (SAS inc., North Carolina, USA), considerando como error alfa 0.05 . 


\section{Resultados}

Los resultados no indicaron efecto significativo $(p>0.05)$ del tratamiento y de la interacción tratamiento por mes de evaluación en la producción de materia seca, sin embargo se observó efecto significativo $(p<0.05)$ del mes de evaluación en esta variable (Figura 1).

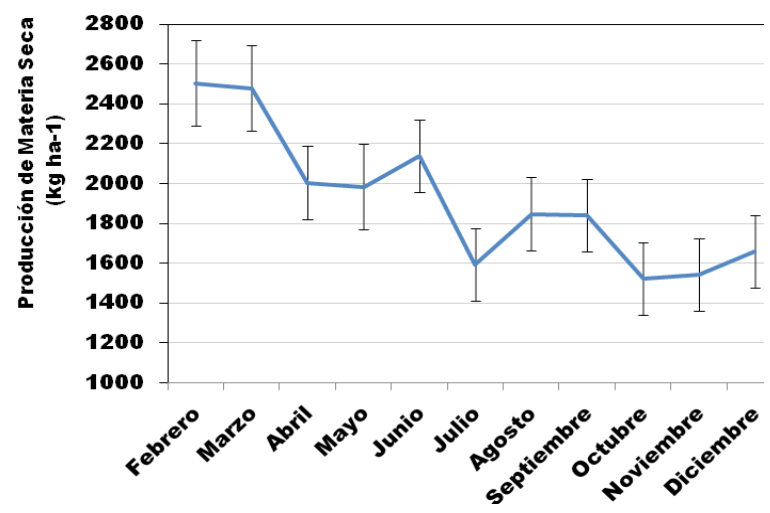

Figura 1. Efecto del tiempo en la producción de materia seca en los tres arreglos silvopastoriles y en la pradera control. Cl Turipaná, Cereté (Córdoba).

En términos de calidad bromatologica se observó efecto significativo $(p<0.05)$ del tratamiento en el porcentaje de proteína cruda (PC) de la gramínea Panicum maximum. El tratamiento p-Arbu-Arbo presentó el mayor registro de PC, con un valor de $12.6 \%$, seguido del tratamiento $\mathrm{p}$-Arbo con $11 \%$, p-Arbus con $10 \%$ y por último el tratamiento sin árboles con un registro de $9.7 \%$ de PC. No se observaron efectos significativos del tratamiento en la proporción de FDN, FDA y DIVMS en la gramínea $P$. maximum (Tabla 2 ).

Tabla 2. Composición bromatológica de P. maximum bajo tres arreglos silvopastoriles y bajo una pradera sin árboles. CI Turipaná, Cereté (Córdoba).

\begin{tabular}{ccccc}
\hline Tratamiento & PC & FDN & FDA & DIVMS \\
\hline Pasto & 9,70 & 78,66 & 43,90 & 54,60 \\
P-Arbu & 10,00 & 78,55 & 43,31 & 56,13 \\
P-Arbo & 11,93 & 79,28 & 44,02 & 57,67 \\
$\begin{array}{c}\text { P-Arbu-Arbo } \\
\text { Error Estándar de la }\end{array}$ & 12,59 & 79,12 & 42,74 & 59,61 \\
Media & 1,49 & 1,07 & 0,72 & 1,80 \\
\hline Valor de p & 0,01 & 0,52 & 0,8 & 0,19 \\
\hline
\end{tabular}

$\mathrm{PC}=$ Proteína Cruda; FDN=Fibra en Detergente Neutro; FDA= Fibra en Detergente Ácido; DIVMS= Digestibilidad de la Materia Seca.
Los datos bromatológicos obtenidos de las especies arbustivas Leucaena leucocephala y Crescentia cujete, en los tratamientos p-Arbust y $p$-Arbust-Arbor, no registraron efecto del tratamiento $(p>0.05)$ en las variables evaluadas (Tabla 3).

Tabla 3. Composición bromatológica de L leucocephala y C. Cujete bajo dos arreglos silvopastoriles. $\mathrm{Cl}$ Turipaná, Cereté (Córdoba)

\begin{tabular}{ccccccccc}
\hline \multirow{2}{*}{ Tratamiento } & \multicolumn{2}{c}{ PC } & \multicolumn{2}{c}{ FDN } & \multicolumn{2}{c}{ FDA } & \multicolumn{2}{c}{ DIVMS } \\
& LI & Cc & LI & Cc & LI & Cc & LI & Cc \\
\hline P-Arbu & 30,25 & 14,58 & 55,81 & 62,43 & 24,01 & 48,36 & 56,82 & 53,77 \\
P-Arbu-Arbo & 32,62 & 15,66 & 52,89 & 63,64 & 23,75 & 52,53 & 65,89 & 50,78 \\
Error Estándar & 1,02 & 0,46 & 2,51 & 1,82 & 3,82 & 1,75 & 2,33 & 1,63 \\
\hline Valor de p & 0,24 & 0,21 & 0,35 & 0,59 & 0,96 & 0,23 & 0,11 & 0,27 \\
\hline
\end{tabular}

$\mathrm{PC}=$ Proteína Cruda; FDN=Fibra en Detergente Neutro; FDA= Fibra en Detergente Ácido; DIVMS= Digestibilidad de la Materia Seca; LI: Leucaena leucocephala, Cc: Crecentia cujete

Los resultados obtenidos en la carga animal registraron efecto del tratamiento, del mes de evaluación y de la interacción entre estos factores. El tratamiento $p$-Arbus registró la mayor carga, con un valor de 3.6 animales ha-1, el cual superó 14, 27 y $39 \%$ los resultados obtenidos en los tratamientos Pasto, p-Arbu-Arbo y p-Arbo, respectivamente (Figura 2).



Figura 2. Carga en animales ha-1, para tres arreglos silvopastoriles y una pradera sin árboles. Cl Turipaná, Cereté (Córdoba) 
La dinámica de la carga en el tiempo evidenció que durante los meses de mayor precipitación (Mayo - Octubre) los tratamientos Pasto y p-Abus registraron los mayores valores de carga animal con oscilaciones entre 3 y 4 animales ha ${ }^{-1}$ para el primero y entre 3 y 5 animales ha ${ }^{-1}$ para el segundo tratamiento. Contrario a esto, las cargas animales en estos tratamientos se vieron afectadas hasta en un $50 \%$ durante el período seco.

Los sistemas silvostoriles p-Arbo y p-ArbuArbo, presentaron una dinámica contraria a la descrita anteriormente. Durante el período seco se observaron cargas más altas en estos tratamientos, con valores que oscilaron entre 2 y 4 animales ha $^{-1}$ para el tratamiento $p$-Arbo y entre 3 y 5 animales ha-1 en el p-Arbu-Arbo (Figura 3 )

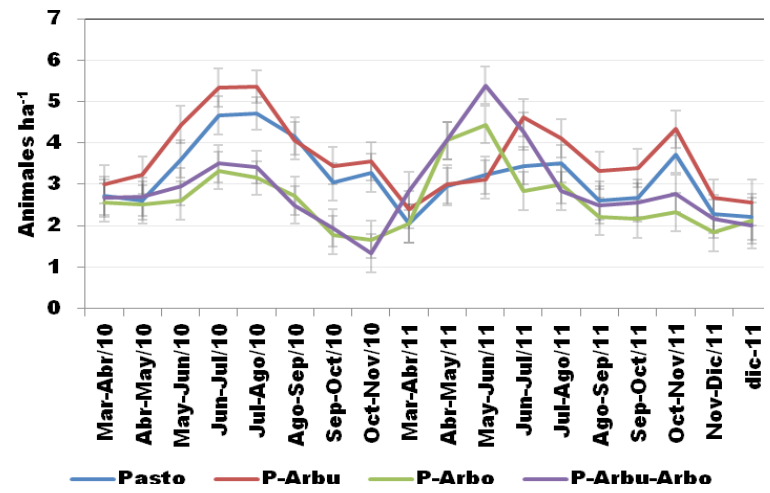

Figura 3. Dinámica de la carga en animales ha-1, para tres arreglos silvopastoriles y una pradera sin árboles. Cl Turipaná, Cereté (Córdoba).

Los resultados obtenidos para el desempeño individual medido a través de la curva de lactancia empleando el modelo de Wood (Figura 4 ), indicaron que el tratamiento no presentó efecto significativo $(p>0.05)$ en el tiempo al pico de lactancia, la producción de leche al pico y producción total en la lactancia (Tabla 4).

La producción de leche por unidad de superficie presentó efecto significativo del tratamiento $(p<0.05)$. Sin embargo, no se observaron efectos $(p>0.05)$ del mes de evaluación ni de la interacción entre el tratamiento y el mes.

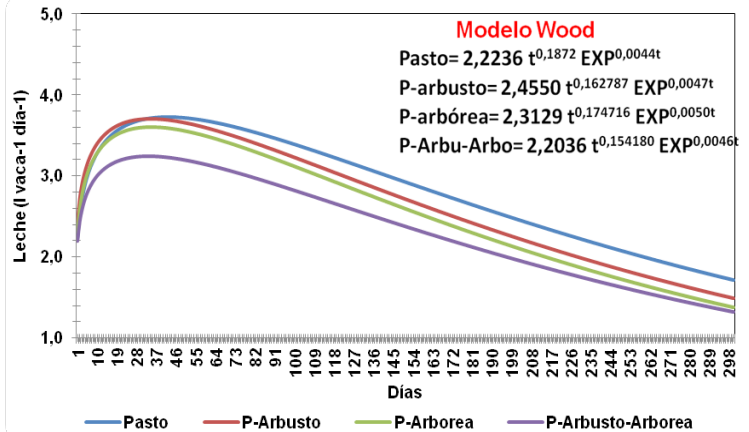

Figura 4. Curva de lactancia aplicando el modelo de Wood para tres sistemas silvopastoriles y una pradera sin árboles. Cl Turipaná, Cereté (Córdoba)

Tabla 4. Tiempo al pico de producción, producción de leche al pico y producción total en la lactancia para tres arreglos silvopastoriles y una pradera sin árboles. $\mathrm{Cl}$ Turipaná, Cereté (Córdoba)

\begin{tabular}{cccc}
\hline Tratamiento & $\begin{array}{c}\text { Tiempo al pico Producción de } \\
\text { de producción } \\
\text { (días) }\end{array}$ & $\begin{array}{c}\text { leche al pico } \\
\text { (litros) }\end{array}$ & $\begin{array}{c}\text { Producción } \\
\text { total en la } \\
\text { lactancia } \\
\text { (litros) }\end{array}$ \\
\hline Pasto & $39,26(13,93)$ & $3,67(0,64)$ & $812,17(121,3)$ \\
P-Arbu & $36,04(11,63)$ & $3,71(0,86)$ & $830,23(282,7)$ \\
P-Arbo & $37,01(14,56)$ & $3,82(1,06)$ & $729,2(208,3)$ \\
P-Arbu-Arbo & $34,98(10,76)$ & $3,29(0,36)$ & $718,6(184,2)$ \\
\hline Valor de p & 0,8574 & 0,8237 & 0,7158 \\
\hline
\end{tabular}

El tratamiento que incluye especies arbustivas presentó la mayor producción de leche por unidad de superficie, alcanzando un valor de 12,8 I ha $^{-1}$. Este registro superó en 3 I la producción observada en el tratamiento sin árboles y 4.3 y $5 \mathrm{I}$ el volumen de leche producido en los tratamientos p-Arbo y p-Arbu-Arbo, respectivamente.

Los resultados obtenidos en la calidad composicional no indicaron efecto del tratamiento $(p>0.05)$ en la modificación de la grasa, proteína o sólidos No Grasos de la leche. La Tabla 5 indica los resultados obtenidos en cada caso. 
Tabla 5. Parámetros composicionales de leche asociada a tres arreglos silvopastoriles y a una pradera sin árboles. Cl Turipaná, Cereté (Córdoba)

\begin{tabular}{ccccccc}
\hline Parámetro & Pasto & P-Arbu & P-Arbo & P-Arbu-Arbo & Error Estándar de la Media & $\boldsymbol{p}$-Valor \\
\hline Proteína & 3,93 & 3,67 & 4,13 & 3,91 & 0,21 & 0,4852 \\
Grasa & 3,91 & 3,85 & 4,19 & 4,11 & 0,35 & 0,8718 \\
Solidos No Grasos & 9,57 & 9,29 & 9,86 & 9,67 & 0,19 & 0,2400 \\
\hline
\end{tabular}

\section{Discusión}

Los resultados indicaron la capacidad de adaptación de la gramínea $P$. maximum a ambientes silvopastoriles y la influencia que ejercen este tipo de sistemas en la modificación de las proporciones proteicas en la composición de la materia seca.

Diferentes estudios han reportado efectos de ambientes silvopastoriles en la reducción de la producción de materia seca para especies del género Brachiaria (VILLANUEVA et al., 2008; PACIULLO et al., 2010) y Panicum (DURR Y RANGEL, 2000; OBISPO et al., 2008; VIAFARA et al., 1997); pese a que ambas son catalogadas como medianamente tolerantes al sombreamiento (WONG, 1991). DURR y RANGEL (2000) afirman, para la especie $P$. máximum que, reducciones en la biomasa de esta gramínea bajo condiciones de sombra, indican que el factor luz es absolutamente limitante para la fotosíntesis y acumulación de carbohidratos, reportando rangos de $12-28 \%$ de luminosidad como críticos. En el presente trabajo no se registraron efectos del tratamiento en la producción de materia seca, lo que puede sugerir que los sistemas silvopastoriles no han limitado la producción de materia seca por competencia de luz.

Las modificaciones bromatológicas de la gramínea $P$. maximum en ambientes bajo sombra han sido reportadas para la cantidad de proteína en la materia seca (WILSON y WILLD, 1991; WONG y WILSON, 1985; BENJAMIN et al., 1991) y en menor medida para parámetros como digestibilidad, FDN y FDA (LADYMAN et al., 2003). Estos cambios en la composición proteica del forraje por efecto de la sombra han sido reportados por el desarrollo de gramíneas bajo árboles leguminosos, los cuales están asociados a mayor ciclaje de nutrientes (aporte de hojarasca) y disponibilidad de nitrógeno en el suelo. (WILSON y WILLD, 1991; TREYDET et al., 2007).
No se reportan estudios que refieran el uso de modelos de curvas de lactancia en ambientes silvopastoriles y mucho menos en animales de las características genéticas a los utilizados en este trabajo. Sin embargo, diferentes autores han reportado el uso de otros modelos para describir la curva de lactancia en bovinos doble propósito, aplicando modelos cuadráticos (OSSA et al., 1997) y Polinomial inverso (BOTERO y VERTEL, 2006) con resultados que logran simular el fenómeno biológico. En este estudio, el modelo de Wood describió el fenómeno biológico de la curva de lactancia en animales de cruces $\mathrm{RHC}$. Sin embargo, no se registraron diferencias estadísticas en las variables evaluadas para cada uno de los tratamientos.

Estudios realizados por CAJAS-GIRÓN (2002) en el mismo sistema silvopastoril sobre el cual se realizó este trabajo, pero usando animales F1 de cruce Holstein x Cebú, reportan producción por hectárea que superan el volumen de leche registrado en el presente trabajo. El mismo autor, reporta producciones de leche en los sistemas silvopastoriles que superan hasta en un $50 \%$ el volumen por hectárea registrado en la pradera sin árboles. Lo anterior sugiere que la producción de leche por hectárea en el presente estudio estuvo limitada por el potencial genético de los animales empleados, los cuales tiene un componente importante de Romosinuano caracterizado como animal para producir carne (MONTES et al, 2009).

Pese a las limitaciones productivas de los animales evaluados para producción de leche, se observó que el sistema silvopastoril que incluyó arbustos, superó significativamente la producción de leche por hectárea registrada en el tratamiento sin árboles. Lo anterior indica que si bien no se tiene animales altamente productivos, se observa un mayor potencial de producción en leche ha-1 día $^{-1}$ en el modelo silvopastoril con especies arbustivas, comparado con el tratamiento control, 
lo que puede estar atribuido a la mejor calidad nutricional de la dieta ofertada.

Estudios realizados por CAJAS-.GIRÓN (2002) indicaron que este modelo silvopastoril produjo $74 \%$ más leche ha- ${ }^{-1}$ día $^{-1}$ durante el periodo seco, atribuyendo este resultado a la participación de las especies arbustivas C. cujete, L. leucocephala y G. sepium en la dieta de los animales medida a través de n-Alkanos, la cual fue del orden del $50 \%$ de la materia seca consumida.

En el presente trabajo no se registraron efectos significativos de los sistemas silvopastoriles sobre la calidad composicional de la leche, sin embargo está referenciado en la literatura que los ambientes silvopastoriles ofertan una dieta diversificada con mayor aporte proteico (CAJASGIRÓN, 2002), lo cual impacta positivamente la calidad composicional de leche (SOUZA 2002; BETANCOURT et al., 2003; HERNÁNDEZ y PONCE, 2004; RONCALLO et al., 2009). La ausencia de efecto significativo sobre la calidad de la leche de las dietas silvopastoriles en el presente trabajo, pudieron estar influenciadas por la características genéticas de las vacas usadas en el estudio, las cuales pudieron limitar la capacidad de la dieta consumida en los cambios de la composición de la leche.

\section{Conclusiones}

Fueron evidentes los efectos de los modelos silvostoriles en la modificación de la calidad proteica de la gramínea Panicum máximum. Sin embargo, las mejoras en la dieta no se reflejaron tangiblemente en el aumento individual de la producción ni en las modificaciones de los componentes nutricionales de la leche.

El modelo silvopastoril que incluye arbustos resultó en mayor eficiencia de la capacidad de producción de leche por unidad de superficie, lo cual pudo estar influenciado una mejor calidad de forraje ofertado y mayor capacidad de carga.

El grupo genético utilizado en el trabajo se constituyó en un limitante para evidenciar las bondades de los sistemas silvopastoriles referenciadas en la literatura y registradas en pasadas experiencias bajo los mismos modelos.

\section{Referencias}

BETANCOURT, K.; IBRAHIM, M.; HARVEY, C.; AND VARGAS, B. 2003. Efecto de la cobertura arbórea sobre el comportamiento animal en fincas ganaderas de doble propósito en Matiguás, Matagalpa, Nicaragua. Agroforestería en las Américas 10(39-40): 47-51.

BOTERO, L.; VERTEL M. 2006. Modelo matemático aplicado a la curva de lactancia en ganado vacuno doble propósito. Revista MVZ, 11(1): 759 - 765.

CAJAS-GIRÓN Y.S 2002 Impacts of tree diversity on the productivity of silvopastoral systems in seasonally dry areas of Colombia. PhD thesis, University of Wales, Bangor. UK. 214 pp.

CAJAS-GIRÓN Y.S.; MARTÍNEZ J. C.; PANZA B. D.; MOGOLLÓN A, BARRAGAN W. A et al. 2009 Valoración integral de los beneficios de Sistemas Silvopastoriles de estratos múltiples sobre la sostenibilidad del suelo y la productividad animal en el Sistema doble propósito de la región Caribe. CORPORACION COLOMBIANA DE INVESTIGACION AGROPECUARIA. 191 p.

DURR, P. A; RANGEL J. 2000. The response of Panicum maximum to a stimulate subcanopy environment. Tropical Grassland 34:110-117.

GARCÍA, F. 2010. Efecto de la cobertura arbórea en potreros y el estado de lactancia, sobre el comportamiento diurno de ganado doble propósito manejado bajo pastoreo en el trópico sub-húmedo. Tesis de Maestría. CATIE. Turrialba, Costa Rica. 115p. 
HERNÁNDEZ, R.; PONCE P. 2004. Efecto del silvopastoreo como sistema sostenible de explotación bovina sobre la composición de la leche. LRRD, 16(6).

HOLDRIDGE, L.R.; GRENKE, W.C.; HATHWAY, W.H.; LIANG, T.; TOSI, J.A. 1971. Forest environments in tropical life zones, a pilot study. Pergamon, Oxford. Pp. 747.

HOYOS, P.; GARCÍA, O.; TORRES, M. I. 1995. Manejo y utilización de pasturas en suelos ácidos de Colombia. Fascículo 4 de la Serie "Capacitación en Tecnología de Producción de Pastos". Centro Internacional de Agricultura Tropical (CIAT). Pp. 120.Cali.

LADYMAN, K.P.; KALLENBACH, R. L.; GARRETT H. E., VAN SAMBEEK, J. W.; NAVARRETE-TINDALL N. E 2003. Quality and Quantity Evaluations of Shade Grown Forages. AFTA 2003 Conf. Proceeding.

MONTES, D.; BARRAGAN, W.; VERGARA, O. 2009. Parametros genèticos de caracterìsticas productivas y reproductivas para ganado tipo carne en Colombia. Rev. Colombiana cienc. Anim. 1(2).p 302 - 318.

NAIR PKR, BURESH. R. J.; MUGENDI, D. N.; LATT C. R. 1999. Nutrient cycling in tropical agroforestry systems: Myths and science. In: Agroforestry in sustainable agricultural systems (Buck LE, Lassoie JP and Fernandes ECM, eds.). Boca Raton, FL., CRC Press pp 1-31.

OBISPO, N. E.; ESPINOZA, Y.; GIL, J. L, OVALLES, F.; RODRIGUEZ, M. F. 2008. Efecto del sombreamiento sobre la producción y calidad del pasto guinea (Panicum máximum) en un sistema silvopastoril. Zootecnia Trop 26:285-288.

OBSERVATORIO DEL CARIBE COLOMBIANO 2006. Indicadores Económicos y .Sociales de la Región Caribe Colombiana

OSSA, G.; TORREGROSA, L.; ALVARADO, L. 1997. Determinación de la curva de lactancia en vacas mestizas de un hato de doble propósito en la Región Caribe de Colombia. Revista CORPOICA 2(1). p $54-57$.

PACIULLO, D. S.; CASTRO, C. R.; GOMIDE, C. A.; FERNANDEZ, P.; ROCHA, W.; MÜLLER, M.; ROSIELLO, R. O. 2010. Soil bulk density and biomass partition of Braciaria decumbens in silvopastoral systems. Sci. Agric 67: 598-603.

PEREZ, J.; MARTíNEZ, G.; ALBARADO, L.; Y OSSA, G. 1998. Características Productivas, Reproductivas y Biofísicas de Cuatro Fincas en el Sistema Doble Propósito en el Departamento de Córdoba. La Investigación Pecuaria.

PEZO, D.; IBRAHIM, M. 1999. Sistemas silvopastoriles. CATIE. 2 ed. Turrialba, CR. 276 p. (Colección Módulos de Enseñanza No. 2).

RONCALLO, B.; HENRIQUEZ, J.; BONILLA, R.; MURILLO, J.; DEL TORO, R. 2009. Evaluación de arreglos agrosilvopastoriles en explotaciones ganaderas de la microrregión Bajo Magdalena. Revista Corpoica 10 (1). p 60-69.

SAS.1992 User's guide: Statistics. Version 9.2. Cary, NC: SAS Institute.

SAUZA, M. H, de. 2002. Contribution of trees to the control of heat stress in dairy cows and the financial viability of livestock farms in humid tropics. Ph.D. Thesis. Turrialba, CR, CATIE. 52, $47 \mathrm{p}$. 
SCHROTH G. 2003. Decomposition and Nutrient Supply from Biomass. G SCHROTH AND F.L. SINCLAIR (Eds.) CABI publishing. ISBN 0-85 199-593-4. 437 pp.

TATIS, R.; BOTERO L. 2005. Genesis y Consolidación del sistema vacuno doble propósito. ASODOBLE, Bogotá, Colombia, $282 \mathrm{p}$.

TREYDET, A.; HEITKONIG, I., PRINS, H.; LUDWIG, F. 2007. Trees improve grass quality for herbivores in African savannas. Perspectives in Plant Ecology, Evolution and Systematics 8. p 197-205.

VIAFARA B, CLAVERO T, ARAUJO-FERBES O. Efecto del sombreamiento de Saman (Pithecelobium saman JACQ. (BENTH)) sobre el desarrollo y crecimiento del pasto guinea. Arch. Latinoam. Prod. Anim. 1997; 5: 39-34.

VILLANUEVA, C.; IBRAHIM M, RIOS, J.; SUAREZ J. 2008. Disponibilidad de Brachiaria brizatha en potreros con diferentes niveles de cobertura arbórea en el trópico subhumedo de Costa Rica. Zootecnia Trop 26:293-296.

WILSON, J. R.; WILD, D. W. M. 1990. Improvement of nitrogen nutrition and grass growth under shading. Forages in plantations crops. ACIAR Proc, (32).

WILSON, J. R. 1996. Shade simulated growth and nitrogen uptake by pasture grasses in a subtropical environment. Australian Journal of Agricultural Research 47:1075-1095.

WONG, C. C 1991. Shade tolerance of tropical forages: A review. In: Shelton HM, Stür WW (ed). Forages for plantation crops. Canaberra: ACIAR 1991. P 64-69.

WOOD, P D P 1967. Algebraic model of the lactation curve in cattle; Nature (Londres) 216: 164 - 165 